\title{
Design of Wideband Dual-Polarized Planar Antenna Using Multimode Concept
}

\author{
Deqiang Yang, Huiling Zeng, Yubo Wen, Meng Zou, and Jin Pan \\ Department of Microwave Engineering, School of Electronic Engineering, \\ University of Electronic Science and Technology of China (UESTC), Chengdu 611731, China
}

Correspondence should be addressed to Huiling Zeng; yidingdong@163.com

Received 30 June 2016; Revised 7 September 2016; Accepted 10 October 2016

Academic Editor: Miguel Ferrando Bataller

Copyright (c) 2016 Deqiang Yang et al. This is an open access article distributed under the Creative Commons Attribution License, which permits unrestricted use, distribution, and reproduction in any medium, provided the original work is properly cited.

A wideband dual-polarized planar antenna is designed and analyzed by using the theory of characteristic modes (TCM). The eigenvalue, eigencurrent, characteristic pattern, and modal weighting coefficient are analyzed to bring physical insight to this kind of antenna. The results demonstrate that there are two modes resonant in the operating band for each polarization, which have been combined to form a wider frequency band. A bandwidth of $60.2 \%(1.72-3.2 \mathrm{GHz})$ for VSWR $<1.5 \mathrm{with}$ high isolation of $32 \mathrm{~dB}$ is achieved simultaneously. The size of the radiator structure is $0.33 \lambda_{0} \times 0.33 \lambda_{0} \times 0.22 \lambda_{0}\left(\lambda_{0}\right.$ refers to the center operating frequency).

\section{Introduction}

Dual-polarized antennas have been widely investigated in wireless communications in past decades [1-3]. To realize dual-polarization, two kinds of antenna can be chosen: patch antenna and cross-dipole antenna. In the design process of dual-polarized patch antenna, most of the reported designs focus on the impedance matching and isolation enhancement because the design is clearly based on cavity model [4].

Various dual-polarized planar antennas which are based on crossed dipole have proved their excellent performance and been reported frequently. In the design of this kind of antenna for dual-polarized application, some performance is studied, such as the bandwidth enhancement, isolation improvement, size-reduction, and planarization design [59]. However, there are still many problems existing in the way to analyze the resonant performance of cross-dipole antenna. Generally, this kind of planar antenna is analyzed by studying the elements of the crossed dipole independently. However there are differences in performance between one polarization of crossed dipole antenna and the same linear polarized dipole. It is because that the planar dipole, a twodimensional structure, is different from the line dipole. The crossed line dipole can be analyzed independently because the currents on one of the dipole are orthogonal with the other in dual-polarized application. In [8], this phenomenon is explained by using concept of coupling between the two crossed dipoles. However, it is difficult to bring physical insight to the resonant phenomenon.

In this paper, theory of characteristic modes (TCM) is applied to analyze dual-polarized planar antenna. The eigenvalue, eigencurrent and characteristic pattern are analyzed without feeding mechanism. In dual-polarized antenna, each polarization has two modes which are forced to resonant in the same frequency band to gain a wider operating band. According to the TCM, the proposed antenna can operate for dual polarization application with a wide bandwidth. Then the antenna is simulated and measured and a good performance has been achieved.

This paper is organized as follows. In Section 2, mode analysis is discussed. Section 3 shows the simulated and measured results. Section 4 is a conclusion for this paper.

\section{Mode Analysis}

The proposed antenna is shown in Figure 1. It is fabricated on a square substrate with loss tangent of 0.02 and relative permittivity $\varepsilon_{r}$ of 4.4 . The substrate has a thickness of $0.8 \mathrm{~mm}$ and is located at about $0.26 \lambda_{0}\left(\lambda_{0}\right.$ refers to the center frequency in vacuum) above the reflector. The antenna can be treated as crossed dipole with a ring around them. One dipole is printed on the front side of a dielectric substrate while the 


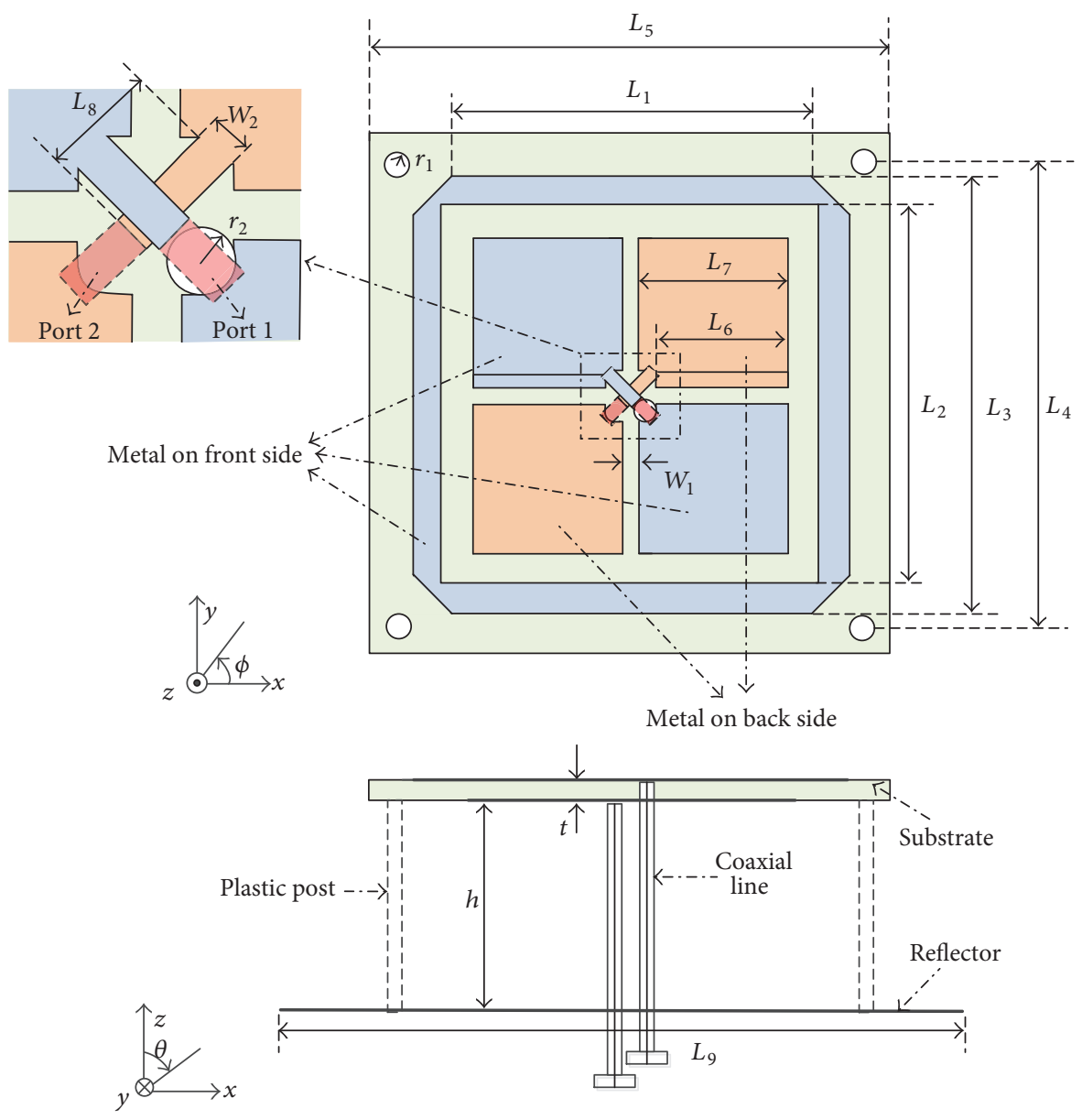

FIGURE 1: Geometry of proposed antenna. Dimensions: $L_{1}=30 \mathrm{~mm}, L_{2}=33.9 \mathrm{~mm}, L_{3}=40.7 \mathrm{~mm}, L_{4}=42 \mathrm{~mm}, L_{5}=47 \mathrm{~mm}, L_{6}=11.7 \mathrm{~mm}$, $L_{7}=13.7 \mathrm{~mm}, L_{8}=2.76 \mathrm{~mm}, L_{9}=100 \mathrm{~mm}, r_{1}=1 \mathrm{~mm}, r_{2}=1 \mathrm{~mm}, W_{1}=0.6 \mathrm{~mm}, W_{2}=1.1 \mathrm{~mm}, t=0.8 \mathrm{~mm}$, and $h=32 \mathrm{~mm}$.

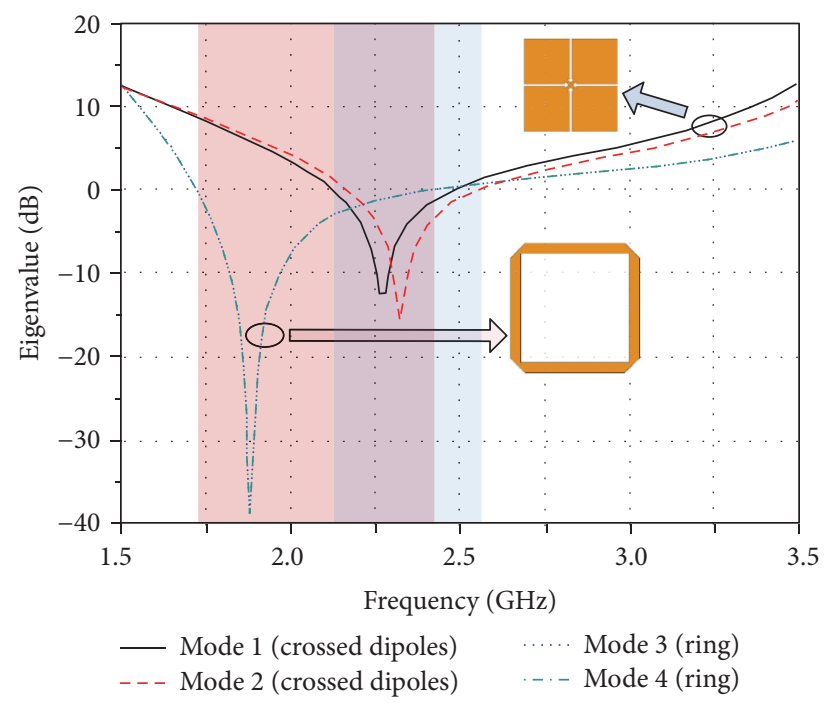

FIGURE 2: Eigenvalues of the crossed dipoles and the ring. (The mode bandwidth can be defined as $\left|\lambda_{n}\right| \leq 0 \mathrm{~dB}$.) 


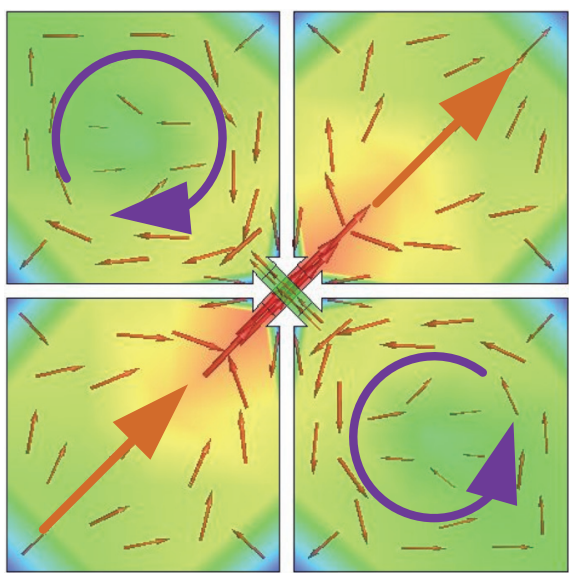

(a)

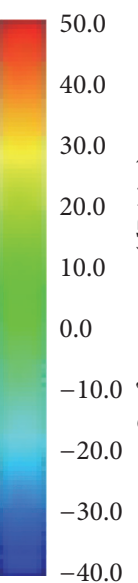

$-40.0$

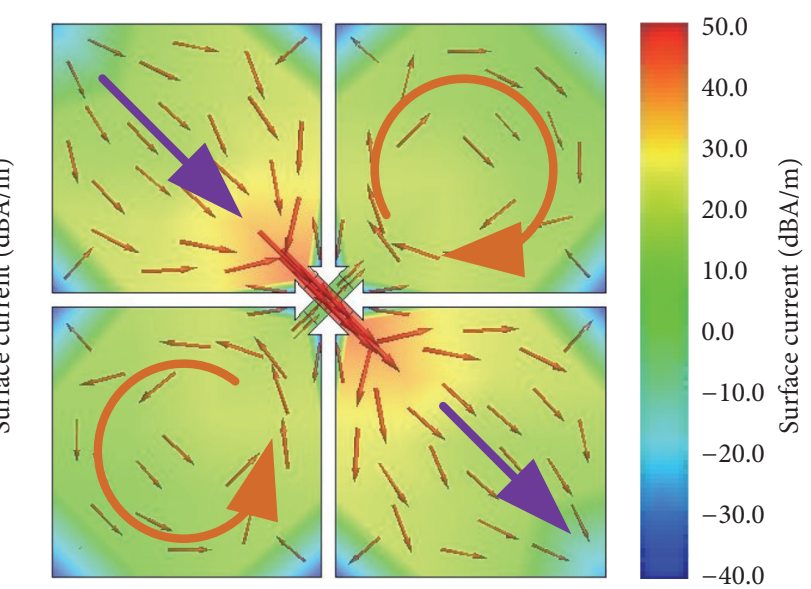

(b)

FIGURE 3: Eigencurrent distribution of the crossed dipoles: (a) mode 1 and (b) mode 2 at their resonance frequency, respectively.

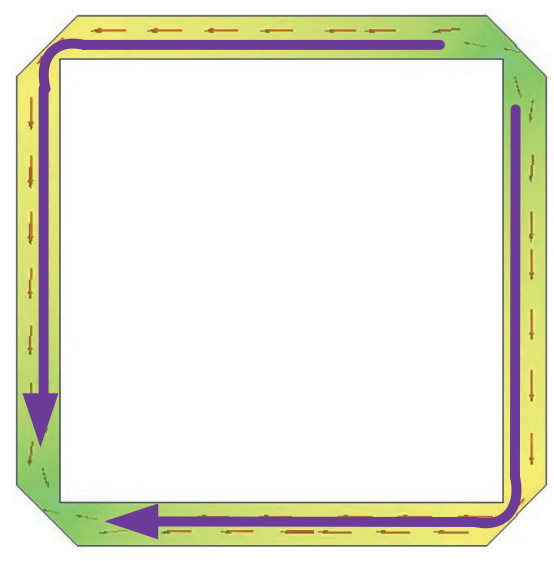

(a)

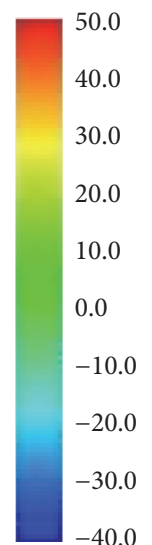

$-40.0$

FIGURE 4: Eigencurrent distribution of the ring: (a) mode 3 and (b) mode 4 at their resonance frequency, respectively.

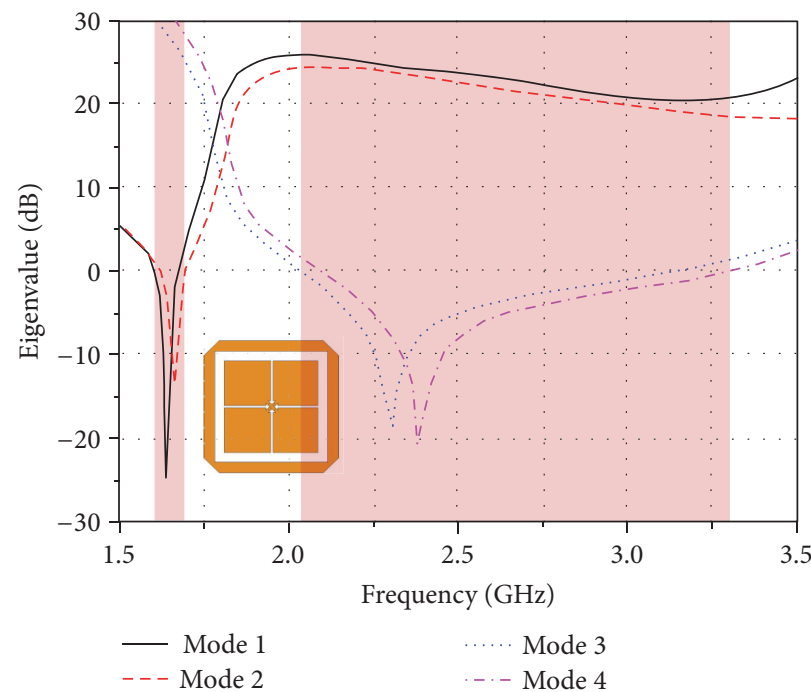

FIGURE 5: Eigenvalue of the proposed antenna. 


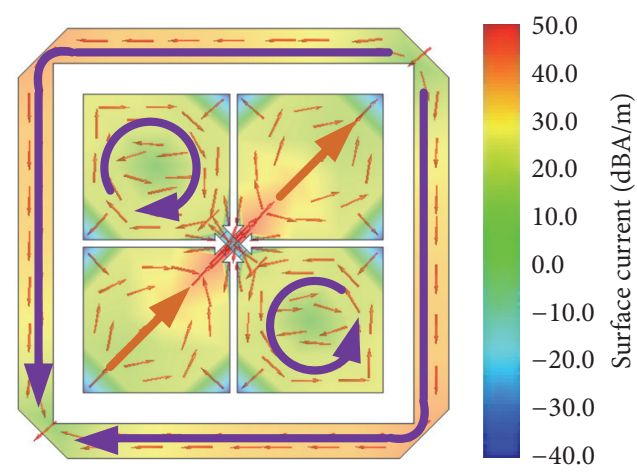

(a)

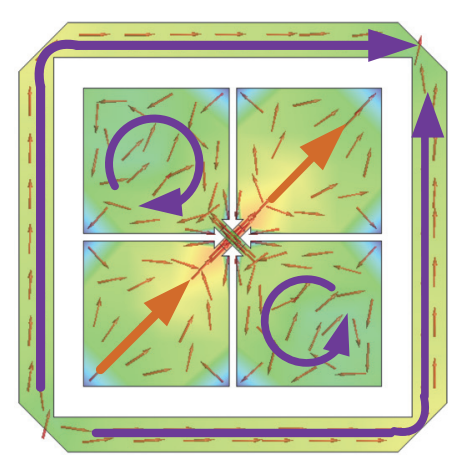

(c)

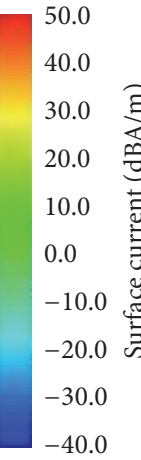

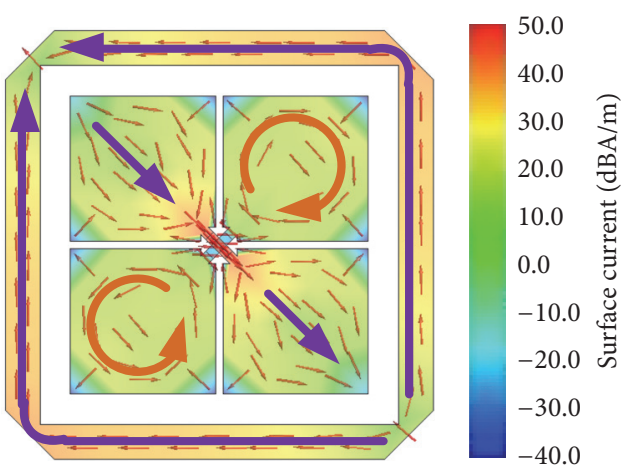

(b)

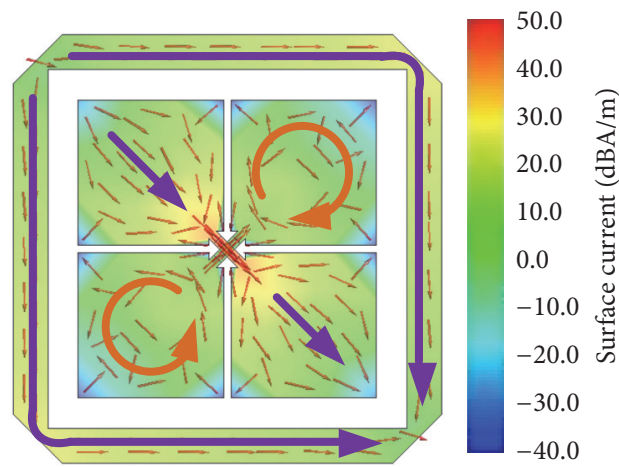

(d)

FIGURE 6: Eigencurrent distribution of the proposed antenna: (a) mode 1, (b) mode 2, (c) mode 3, and (d) mode 4 at their resonance frequency, respectively.

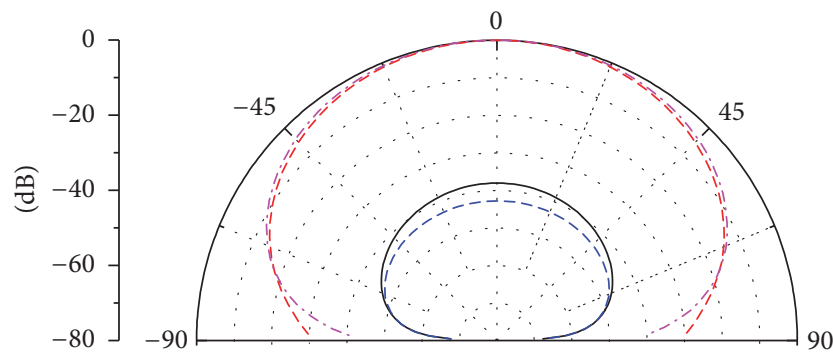

$-E_{\phi}$, mode 1
$---E_{\theta}$, mode 1

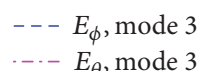

(a1) $\phi=45^{\circ}$

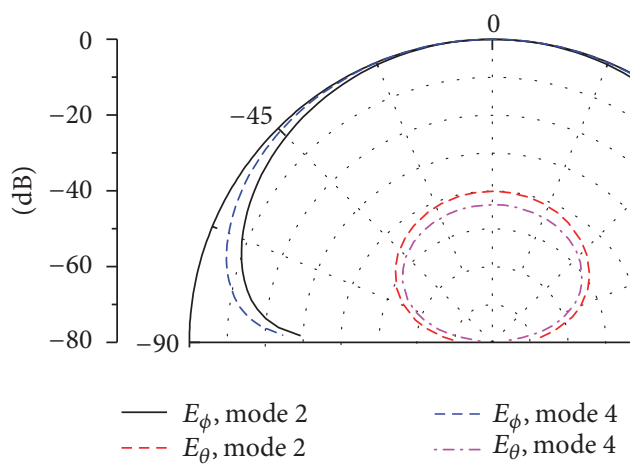

(b1) $\phi=45^{\circ}$
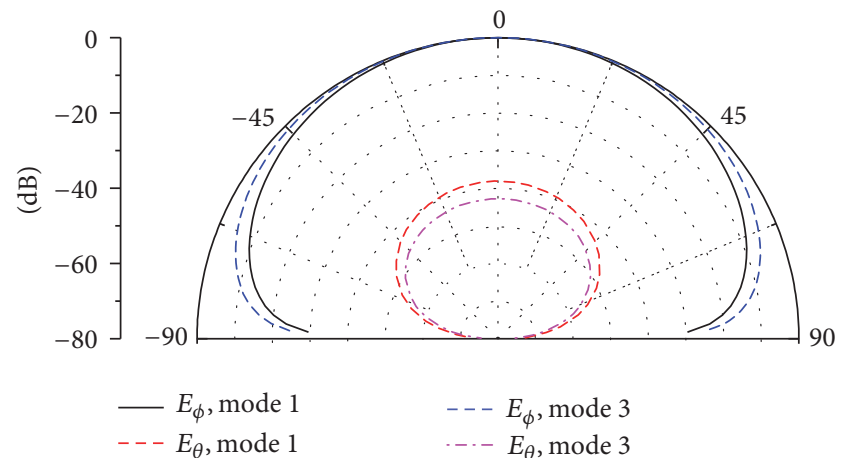

(a2) $\phi=135^{\circ}$

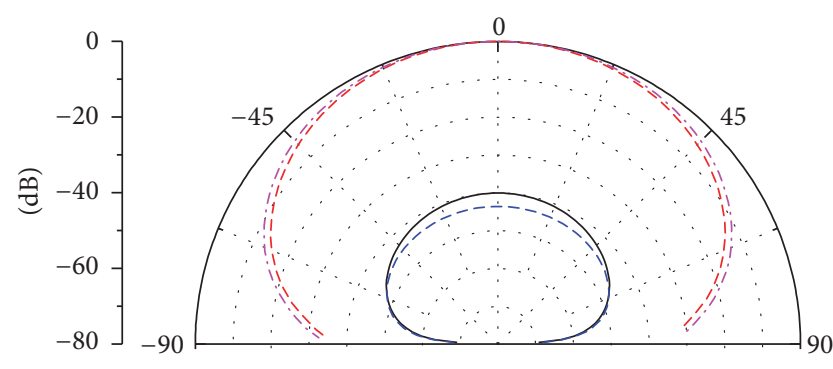

$-E_{\phi}$, mode 2
$--E_{\theta}$, mode 2

- - $E_{\phi}$, mode 4

...- $E_{\theta}$, mode 4

(b2) $\phi=135^{\circ}$

FIgURE 7: Far E-field of each mode of the proposed antenna. 


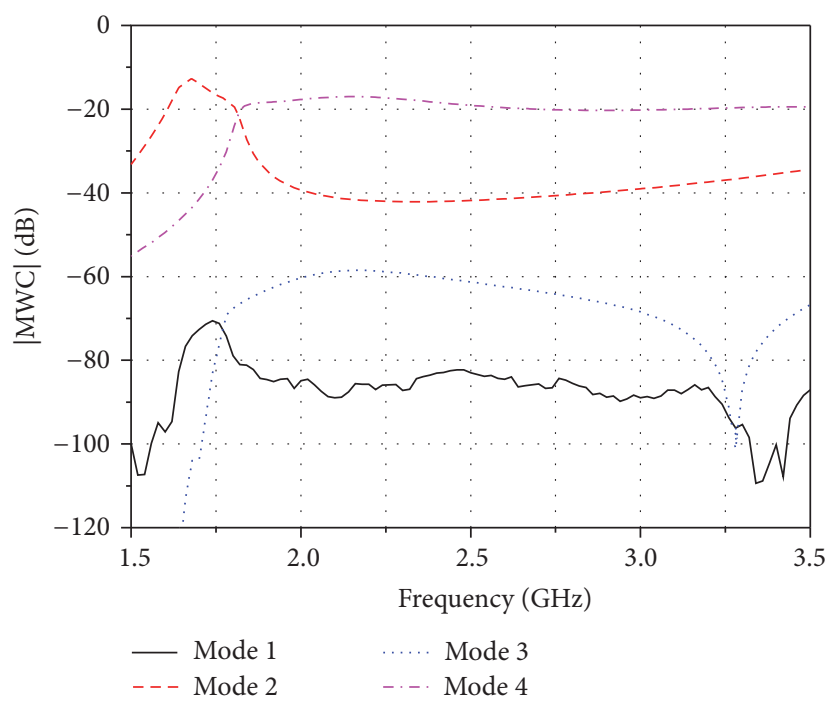

FIGURE 8: Magnitude of MWC in dB of the proposed antenna.

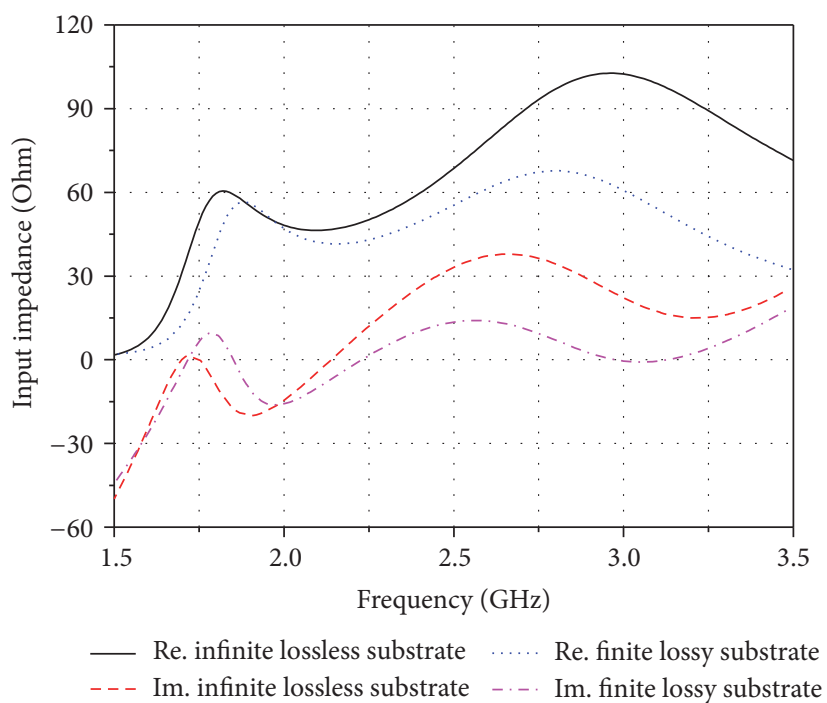

FIGURE 9: Input impedance of the proposed antenna.

other one is etched on the backside. Four plastic screws are employed to support the dual-polarized antenna.

In this section, the characteristic modes of the proposed dual-polarized planar antenna have been analyzed in FEKO [10]. The detail of TCM can be found in [11]. It is important to point out that, in the analysis of characteristic modes, the reflector and the substrate are assumed to be infinite in the transverse directions. The substrate is assumed to be lossless simultaneously.

2.1. TCM Analysis. In this part, the TCM analysis is carried out for the ring, the crossed dipole and the crossed dipole around by ring, respectively.

Eigenvalues of the crossed dipole and the ring with the same dimensions listed in Figure 1 are indicated in Figure 2. Eigencurrents of these resonant modes are shown in Figures 3 and 4 . For the ring, there are two degenerated modes which resonate at about $1.88 \mathrm{GHz}$, while for the crossed dipole, the two degenerated modes resonant at about $2.3 \mathrm{GHz}$, which is the well-known modes used in dual-polarization application.

The eigenvalues in $\mathrm{dB}$ of the proposed antenna are shown in Figure 5. It is found that there are two resonant modes in the band for each polarization. The slight difference on eigenvalue between each polarization is due to the substrate and numerical error. Different with the ring and the crossed dipole, the resonant frequency of the proposed antenna predicted by TCM is about $1.65 \mathrm{GHz}$ and $2.35 \mathrm{GHz}$. And the bandwidth of each mode is different with modes of the ring or crossed dipole. However, the bandwidth of the modes resonant at higher frequency of the proposed antenna is wider than all of the modes of the ring and crossed dipole.

Eigencurrents of the four modes of proposed antenna are shown in Figure 6. For mode 2 (mode 1), the currents on the loop are at the opposite direction with mode 4 (mode 


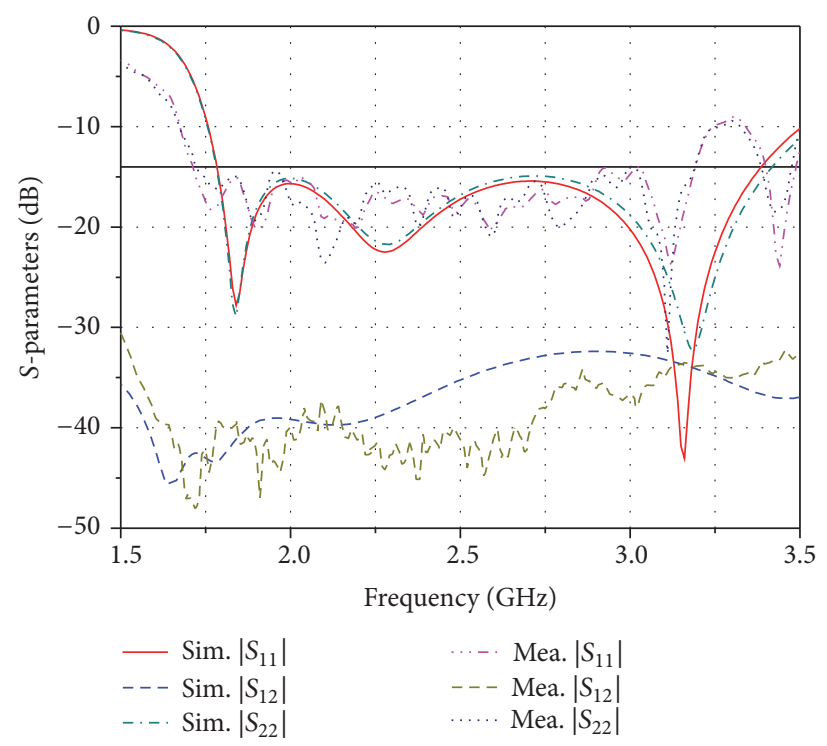

FIGURE 10: Simulated and measured $S$-parameters of the proposed antenna.

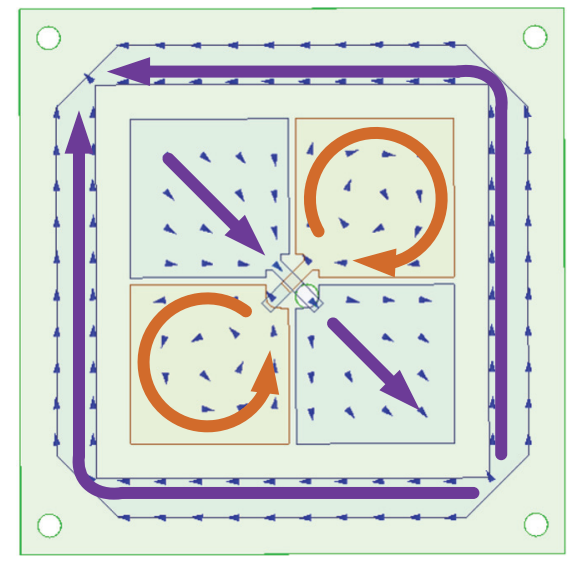

(a)

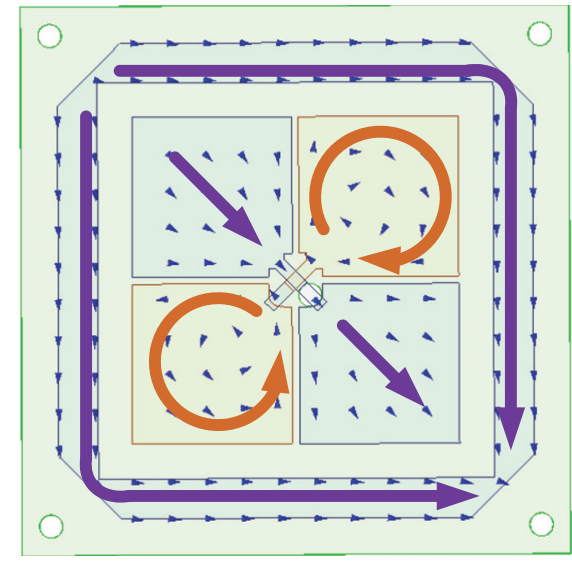

(b)

Figure 11: Current distribution for proposed antenna: (a) $1.72 \mathrm{GHz}$ and (b) $2.23 \mathrm{GHz}$.

3) at their resonance frequency, respectively, while they have similar currents distribution on the inner part. For a crossed dipole, the currents peak is at the middle of the antenna which indicates that these modes can be fed by voltage sources at this position. By exciting in the center of the antenna, these two modes for each polarization shown in Figure 5 can be combined to widen the bandwidth after examining the radiation patterns of each mode. The analysis of the antenna with excitation is discussed in Sections 2.2 and 3.2.

The normalized radiation patterns of these modes at their resonant frequency are shown in Figure 7. It can be found that the radiation patterns of these four modes are similar with dipole, which can be excited to realize dual-polarization as expected.

2.2. Modal Weighting Coefficient. A modal solution for the current on conducting body can be achieved by using the eigencurrents [11]. Modal weighting coefficient (MWC) can be computed as follows.

$$
\mathrm{MWC}=\frac{V_{n}^{i}}{1+j \lambda_{n}},
$$

where $V_{n}^{i}=\left\langle\mathbf{J}_{n}, \mathbf{E}^{i}\right\rangle=\oiint_{S} \mathbf{J}_{n} \cdot \mathbf{E}^{i} d s, \mathbf{J}_{n}$ is the eigencurrent, $\lambda_{n}$ is the eigenvalue, and $\mathbf{E}^{i}$ is the tangential component of impressed electric field on surface $S$. The edge port is defined at the center of the crossed dipole and voltage source is added on port 1 shown in Figure 1. Depending on the magnitude of MWC shown in Figure 8, mode 2 and mode 4 occupy significant weight in low frequency and high frequency, indicating that they paid major role in radiation. At about $1.8 \mathrm{GHz}, \mathrm{MWC}$ of mode 2 and mode 4 is equal. At lower band, mode 2 plays a significant role, while at higher frequency band, MWC of mode 4 is largest. The modes of the other 


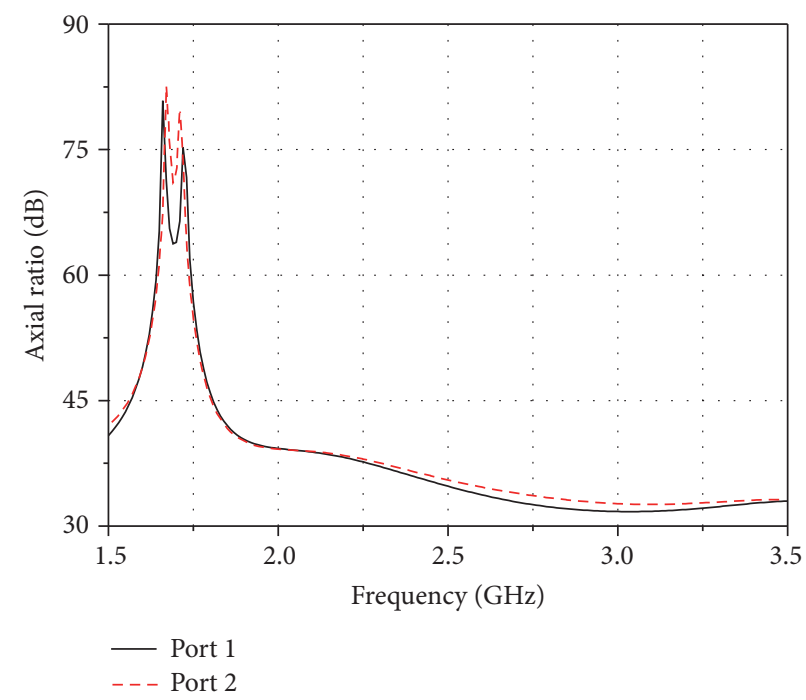

Figure 12: Axial ratio of the proposed antenna.

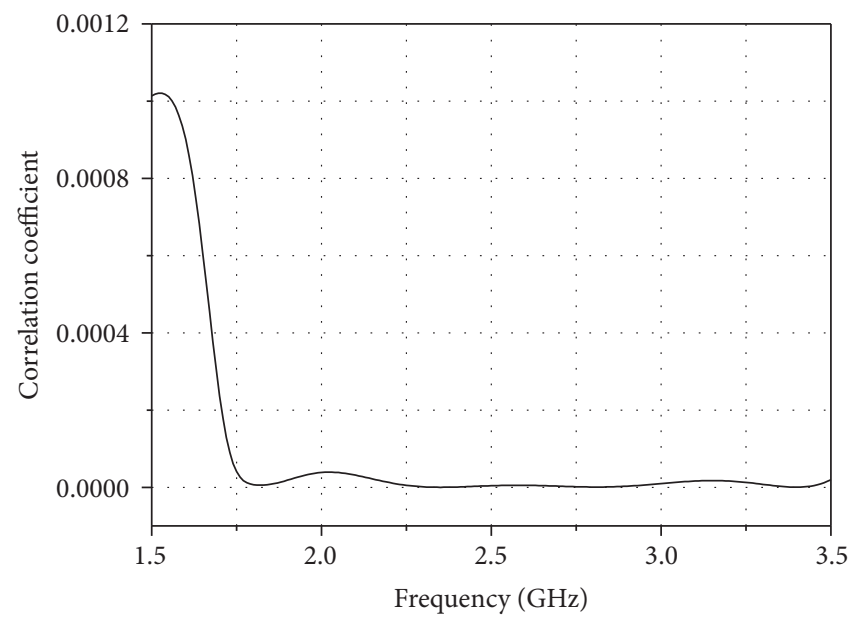

FIGURE 13: Correlation coefficient between the two ports.

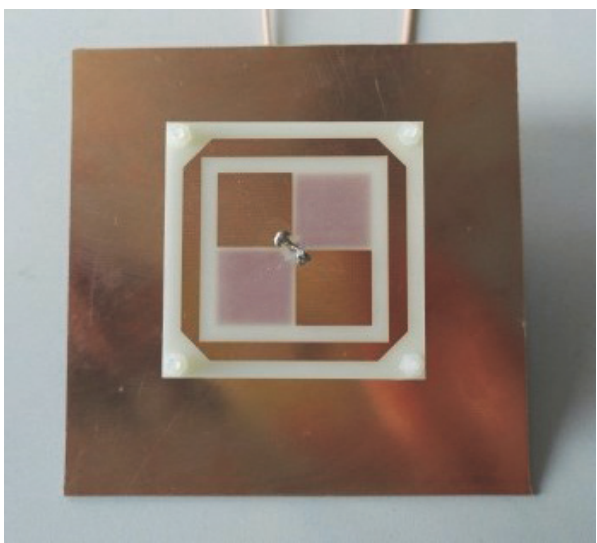

(a)

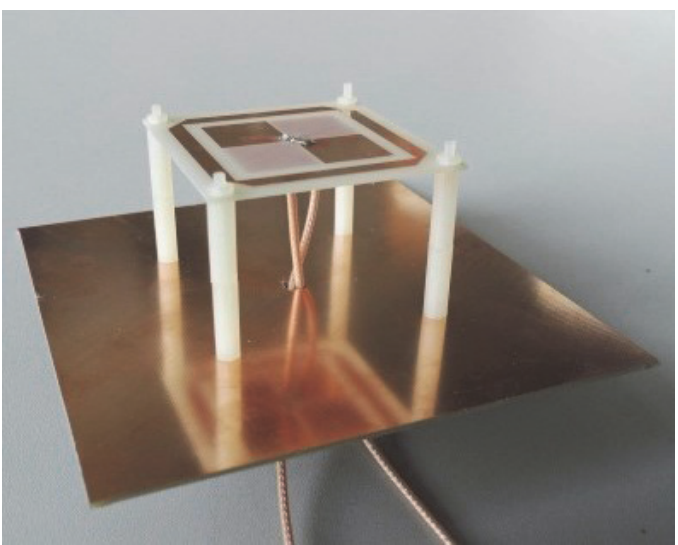

(b)

FIGURE 14: Fabricated prototype proposed antenna: (a) top view and (b) perspective view. 


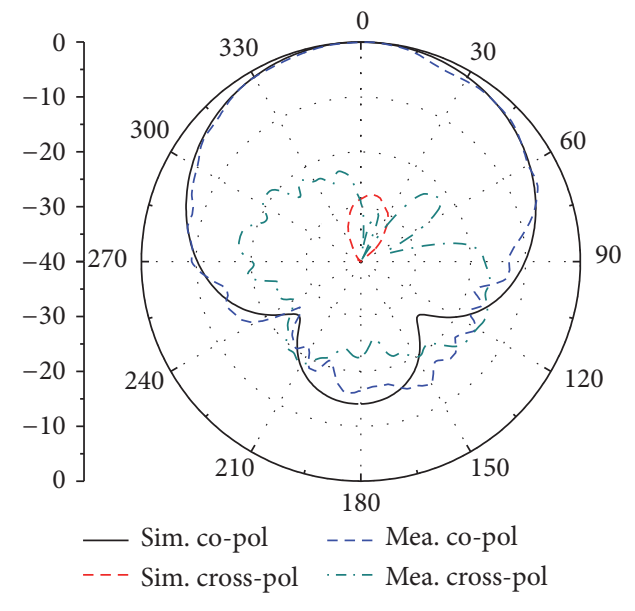

(a1) $\phi=45^{\circ}$

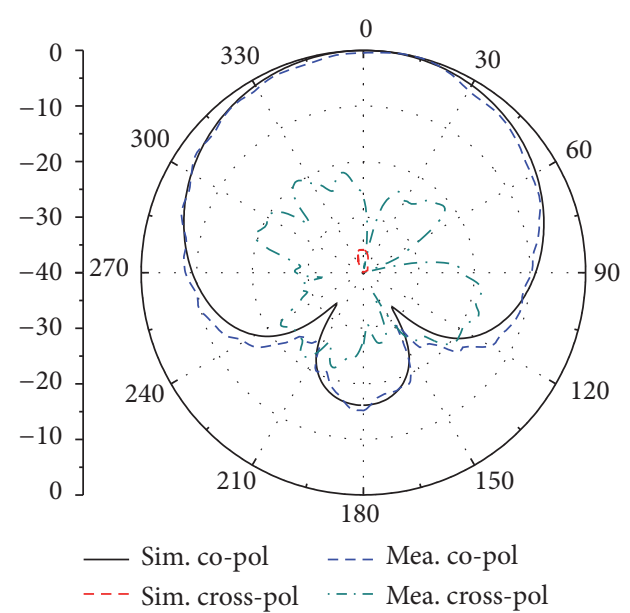

(b1) $\phi=45^{\circ}$

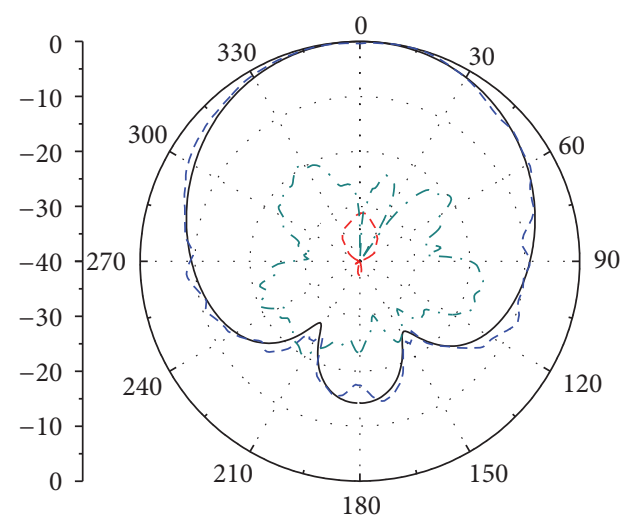

$$
\begin{array}{ll}
\text { _ Sim. co-pol } & \text { - - - Mea. co-pol } \\
\text { _ - - Sim. cross-pol } & \text { _. - Mea. cross-pol }
\end{array}
$$

(c1) $\phi=45^{\circ}$

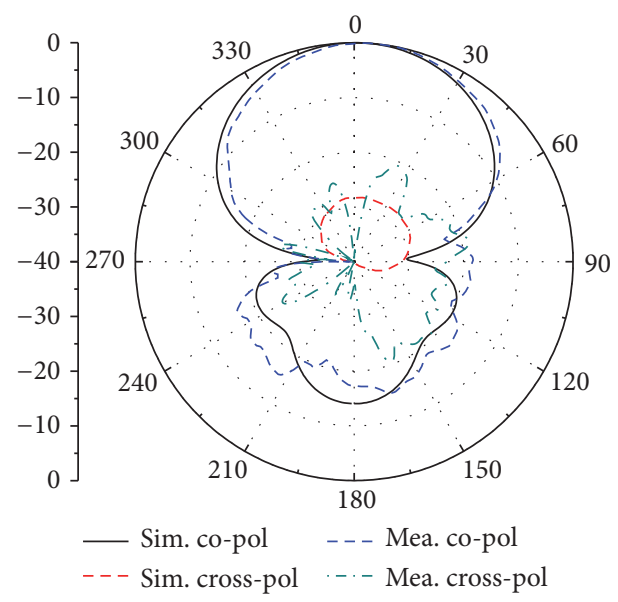

(a2) $\phi=-45^{\circ}$

(a)

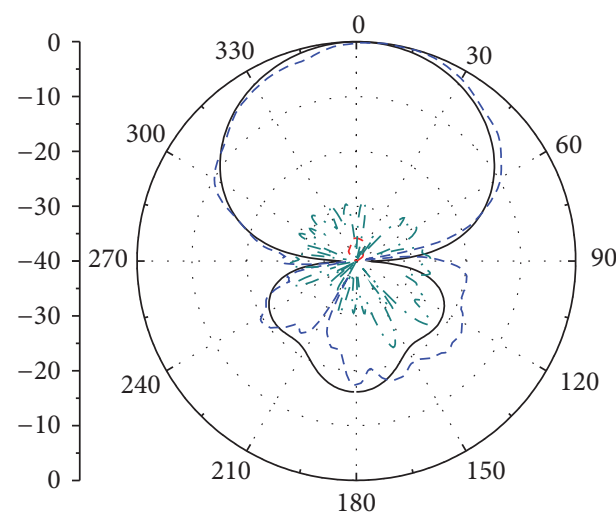

$$
\begin{array}{ll}
\text { — Sim. co-pol } & \text {-- } \text { Mea. co-pol } \\
\text { - - Sim. cross-pol } & \text { - - - Mea. cross-pol }
\end{array}
$$$$
\text { (b2) } \phi=-45^{\circ}
$$

(b)

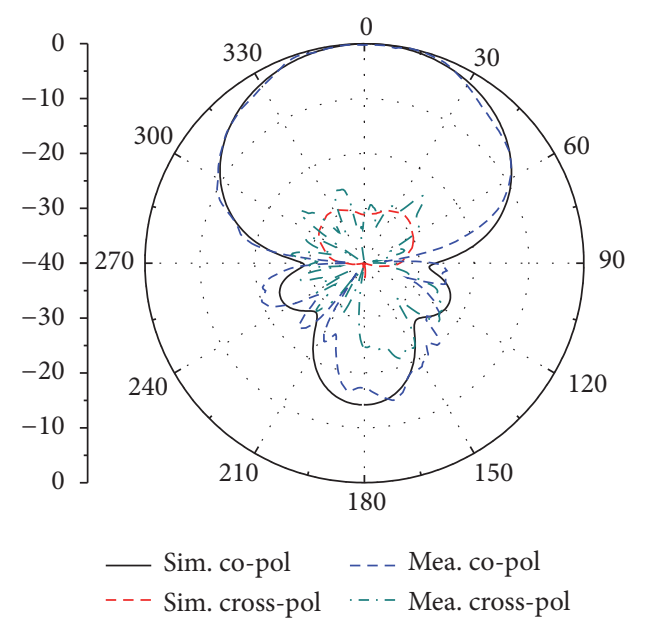

$$
\text { (c2) } \phi=-45^{\circ}
$$

(c)

FIGURE 15: Simulated and measured radiation patterns of the proposed antenna at (a) $1.9 \mathrm{GHz}$, (b) $2.45 \mathrm{GHz}$, and (c) $3 \mathrm{GHz}$. 


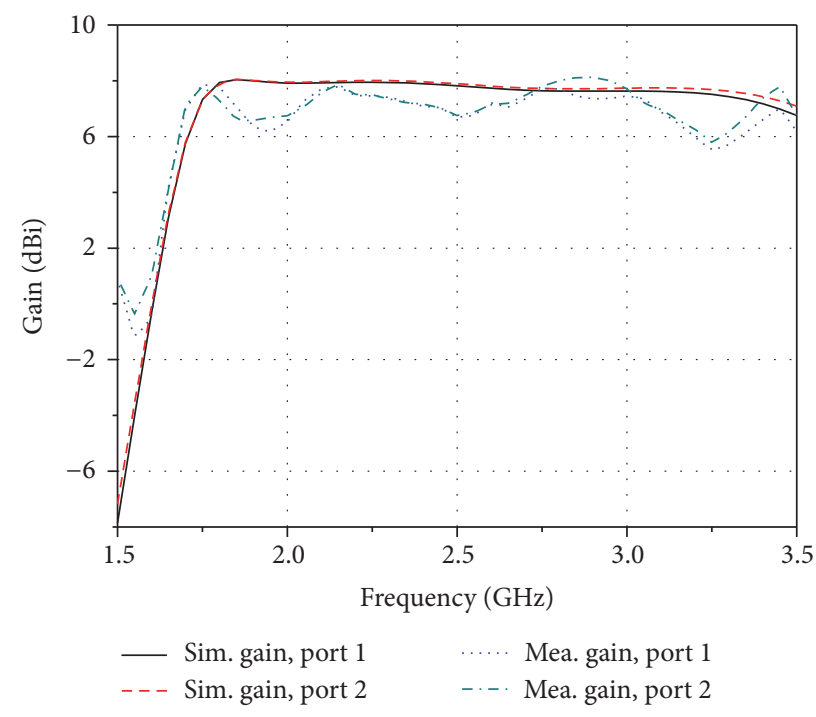

FIGURE 16: Simulated and measured gain of the proposed antenna.

polarization are not excited according to Figure 8. Despite of these four modes, other modes are eliminated in the figure for their small MWC.

\section{Antenna Design}

3.1. Simulated Results. The proposed antenna is simulated in HFSS [12]. Different with the model in analysis of TCM, the antenna analysis in HFSS is fabricated on the lossy substrate with finite size. The input impedance of the antenna with different mediums, infinite lossless substrate and finite lossy substrate, are shown in Figure 6. When the imaginary is part of the input impedance, which indicates that the net stored energy is zero, the phenomenon is called antenna resonance. At about $1.72 \mathrm{GHz}$ and $2.25 \mathrm{GHz}$, the antenna resonance occurred, resulting from the resonance of mode 2 and mode 4. According to the simulated input impedance shown in Figure 9, the substrate has limited effect on resonant frequency.

The simulated $S$-parameters are shown in Figure 10. It is found that a bandwidth of $62.4 \%(1.78-3.39 \mathrm{GHz})$ for VSWR $<1.5$ and high port-to-port isolation of $30 \mathrm{~dB}$ can be achieved. The simulated current distribution on the antenna is shown in Figure 11 which is similar to the results predicted by TCM shown in Figure 6.

The axial ratio (AR) of the two ports is shown in Figure 12. Across the bandwidth, the AR is higher than $30 \mathrm{~dB}$, indicating that a good dual linear polarized radiation performance is achieved at axial direction. The correlation coefficient between the two ports calculated by using simulated 3D radiation pattern in HFSS is shown in Figure 13. The result is nearly zeros which indicated that the proposed antenna can radiate orthogonal fields with polarization diversity.

3.2. Experimental Results. Figure 14 shows the fabricated prototype of the proposed antenna. At Port 1, the coaxial cable goes through the hole and the inner and outer conductor is soldered on each half of dipole printed on the front side of the substrate. At port 2, the coaxial cable is soldered on each half of dipole printed on the back side.

Figure 10 indicates the simulated and measured $S$ parameters of the designed antenna. The operating band moves slightly down to the lower frequency band compared with simulated results which is attributed to the feeding mechanism using inner and outer conductor of coaxial cable. The proposed antenna achieves a $14 \mathrm{~dB}$ return loss bandwidth of $1.72-3.2 \mathrm{GHz}$ for port 1 and port 2 .

Figure 15 shows the radiation patterns simulated and measured at $1.9 \mathrm{GHz}, 2.45 \mathrm{GHz}$, and $3 \mathrm{GHz}$ for $-45^{\circ}$ polarization (The radiation patterns for $+45^{\circ}$ polarization are similar because of geometric symmetry.) The values are normalized by the boresight gain of each frequency, respectively. It points out that the proposed antenna has stable radiation patterns. The measured radiation patterns and simulated ones are in good agreement with each other. Figure 15 shows that the cross-polarization within the main lobe remains less than $-25 \mathrm{~dB}$ and the front-back ratio remains about $14 \mathrm{~dB}$. Figure 16 shows that the measured boresight gain of the antenna is about $7 \mathrm{dBi}$ across the operating band. A comparison between the reference and proposed antennas is listed in Table 1 .

The difference between simulated and measured $S$-parameters and gains results from the fabrication error, lossy substrate, and the feeding mechanism. For simulation in HFSS, ports are added in the middle of the crossed dipole which is aimed at keeping the antenna the same as in mode analysis. Therefore, the antenna's lack of feeding balun for the sake of brevity in mode analysis also leads to the error.

\section{Conclusion}

This paper has analyzed wideband planar dual-polarized antenna based on multimode concept. By inserting a loop for 
TABLE 1: Comparison between the reference and proposed antennas.

\begin{tabular}{|c|c|c|c|}
\hline Ref. & Bandwidth \% & $\begin{array}{l}\text { Radiator size } \\
\left(\mathrm{mm}^{2}\right)\end{array}$ & $\begin{array}{l}\text { Reflector } \\
\left(\mathrm{mm}^{2}\right)\end{array}$ \\
\hline [2] & $\begin{array}{c}45(\text { SWR }< \\
1.5)\end{array}$ & $\begin{array}{c}56 * \\
56\left(0.42 \lambda_{0} *\right. \\
\left.0.42 \lambda_{0}\right)\end{array}$ & $\begin{array}{c}134 * 134 \\
\text { planar }\end{array}$ \\
\hline [3] & $\begin{array}{c}48.2(\mathrm{SWR}< \\
1.5)\end{array}$ & $\begin{array}{c}50 * \\
50\left(0.37 \lambda_{0} *\right. \\
\left.0.37 \lambda_{0}\right)\end{array}$ & $\begin{array}{c}130 * 130 \\
\text { planar }\end{array}$ \\
\hline [4] & $\begin{array}{c}22.7(\mathrm{SWR}< \\
1.5)\end{array}$ & $\begin{array}{c}210 * \\
210\left(0.45 \lambda_{0} *\right. \\
\left.0.45 \lambda_{0}\right)\end{array}$ & Planar \\
\hline [5] & $\begin{array}{c}65.9(\mathrm{SWR}< \\
2)\end{array}$ & $\begin{array}{c}64.6 * \\
64.6\left(0.55 \lambda_{0} *\right. \\
\left.0.55 \lambda_{0}\right)\end{array}$ & $\begin{array}{c}149.6 * 149.6 \\
\text { nonplanar }\end{array}$ \\
\hline [6] & $\begin{array}{c}57.5(\mathrm{SWR}< \\
1.5)\end{array}$ & $\begin{array}{c}49.7 * \\
49.7\left(0.54 \lambda_{0} *\right. \\
\left.0.54 \lambda_{0}\right)\end{array}$ & $\begin{array}{l}300 * 145 \\
\text { nonplanar }\end{array}$ \\
\hline [7] & $\begin{array}{c}48(\mathrm{SWR}< \\
1.5)\end{array}$ & $\begin{array}{c}60.5 * \\
60.5\left(0.45 \lambda_{0} *\right. \\
\left.0.45 \lambda_{0}\right)\end{array}$ & $\begin{array}{l}110 * 110 \\
\text { nonplanar }\end{array}$ \\
\hline Proposed & $\begin{array}{c}60.2(\text { SWR }< \\
1.5)\end{array}$ & $\begin{array}{c}40.7 * \\
40.7\left(0.33 \lambda_{0} *\right. \\
\left.0.33 \lambda_{0}\right)\end{array}$ & $\begin{array}{l}100 * 100 \\
\text { planar }\end{array}$ \\
\hline
\end{tabular}

crossed dipole antenna, two modes are forced to resonate in the frequency band. Both of the modes can radiate a dipolelike pattern which has been used to form a wide operating band. The proposed dual-polarization antenna achieves impedance bandwidth range from $1.72 \mathrm{GHz}$ to $3.2 \mathrm{GHz}$ $(60.2 \%)$ with an isolation higher than $32 \mathrm{~dB}$. In addition, the compact size of about $0.33 \lambda_{0}$ makes the proposed antenna a good candidate for array application.

\section{Competing Interests}

The authors declare that there is no conflict of interests regarding the publication of this paper.

\section{References}

[1] S. X. Ta, I. Park, and R. W. Ziolkowski, "Crossed dipole antennas: a review," IEEE Antennas \& Propagation Magazine, vol. 57, no. 5, pp. 107-122, 2015.

[2] J. A. Kasemodel and J. L. Volakis, "A planar dual linear-polarized antenna with integrated balun," IEEE Antennas and Wireless Propagation Letters, vol. 9, pp. 787-790, 2010.

[3] Y.-H. Huang, Q. Wu, and Q.-Z. Liu, "Broadband dual-polarised antenna with high isolation for wireless communication," Electronics Letters, vol. 45, no. 14, pp. 714-715, 2009.

[4] K. F. Lee and K. M. Luk, Microstrip Patch Antennas, Imperial College Press, London, UK, 2010.

[5] Y. H. Cui, R. L. Li, and H. Z. Fu, "A broadband dual-polarized planar antenna for 2G/3G/LTE base stations," IEEE Transactions on Antennas and Propagation, vol. 62, no. 9, pp. 4836-4840, 2014.

[6] S.-G. Zhou, P.-K. Tan, and T.-H. Chio, "Low-profile, wideband dual-polarized antenna with high isolation and low cross polarization," IEEE Antennas and Wireless Propagation Letters, vol. 11, pp. 1032-1035, 2012.

[7] B. Q. Wu and K.-M. Luk, "A broadband dual-polarized magneto-electric dipole antenna with simple feeds," IEEE Antennas and Wireless Propagation Letters, vol. 8, pp. 60-63, 2009.

[8] Z. D. Bao, Z. P. Nie, and X. Z. Zong, "A novel broadband dualpolarization antenna utilizing strong mutual coupling," IEEE Transactions on Antennas and Propagation, vol. 62, no. 1, pp. 450-454, 2014.

[9] M. Li and K.-M. Luk, "Wideband magnetoelectric dipole antennas with dual polarization and circular polarization," IEEE Antennas and Propagation Magazine, vol. 57, no. 1, pp. 110-119, 2015.

[10] http://www.feko.info/.

[11] R. F. Harrington and J. R. Mautz, "Theory of characteristic modes for conducting bodies," IEEE Transactions on Antennas and Propagation, vol. 19, no. 5, pp. 622-628, 1971.

[12] http://www.ansys.com/. 


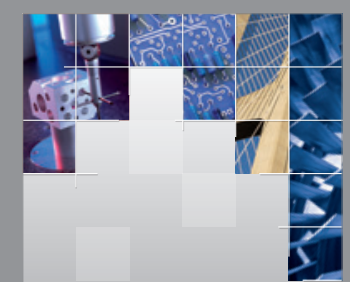

\section{Enfincering}
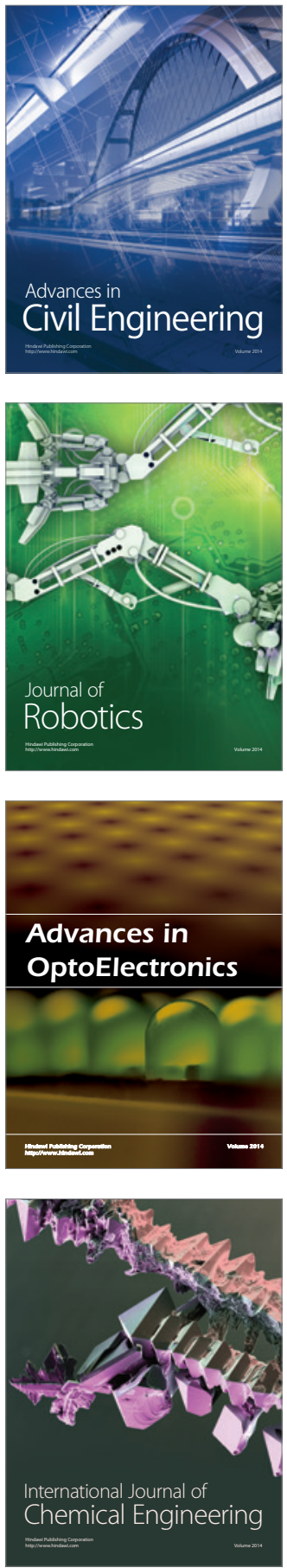

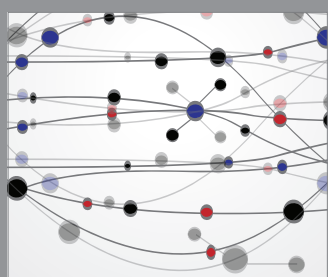

The Scientific World Journal

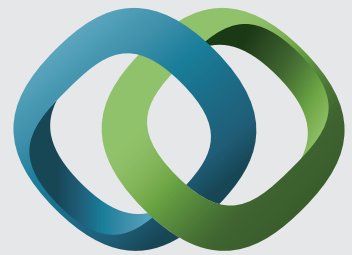

\section{Hindawi}

Submit your manuscripts at

http://www.hindawi.com
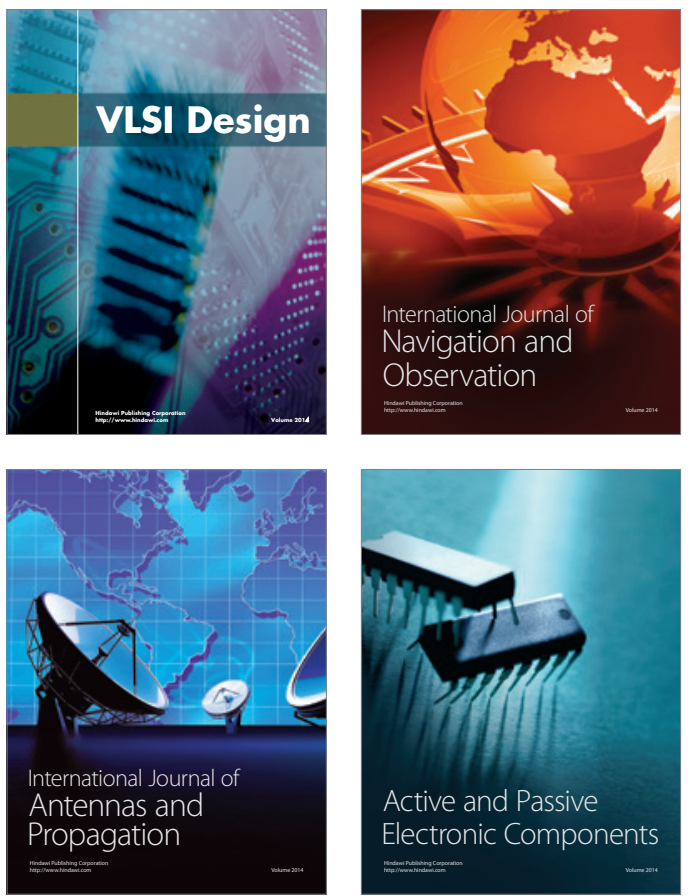
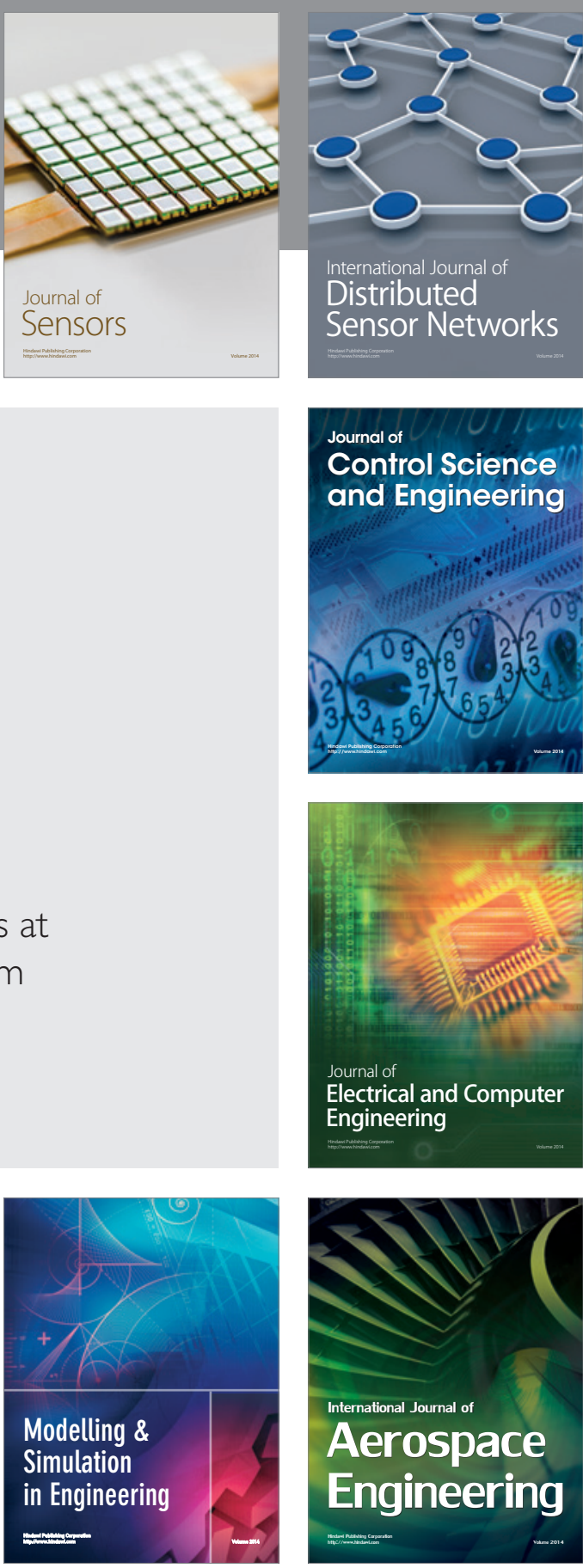

International Journal of

Distributed

Sensor Networks

Journal of

Control Science

and Engineering
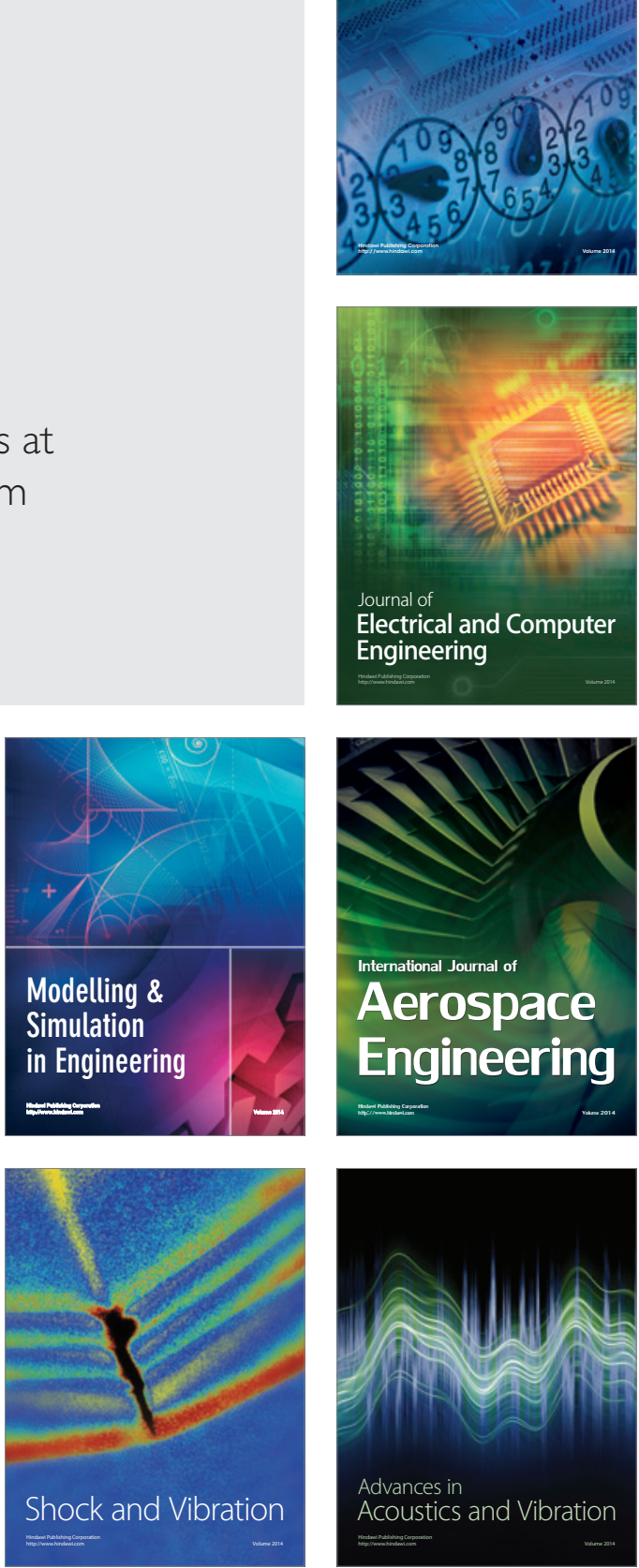